\title{
A Possible Realization of Chlorophyll Laser
}

\author{
Mitali Konwar $^{1}$, G. D. Baruah ${ }^{2}$ \\ ${ }^{1}$ Digboi Mahila Mahavidalaya, Digboi, India \\ ${ }^{2}$ Centre for Laser \& Optical Science, New Uchamati, Doomdooma, India \\ Email: mitalikonwar@rediffmail.com
}

Received October 21, 2013; revised November 23, 2013; accepted December 22, 2013

Copyright (C 2013 Mitali Konwar, G. D. Baruah. This is an open access article distributed under the Creative Commons Attribution License, which permits unrestricted use, distribution, and reproduction in any medium, provided the original work is properly cited. In accordance of the Creative Commons Attribution License all Copyrights (C) 2013 are reserved for SCIRP and the owner of the intellectual property Mitali Konwar, G. D. Baruah. All Copyright @ 2013 are guarded by law and by SCIRP as a guardian.

\begin{abstract}
The present work is concerned with a description of strong fluorescence from a sample of Alocasia culcullata schot (local name Kolia kasu) and possible realization of chlorophyll laser. The red florescence is most prominent when excited with broad band source like $30 \mathrm{~mW}$ green or blue solid state laser, but the acetone extract of plant leaves Allocasa cullcuta schot unusually exhibits the strong intensity in the red sector of the spectrum. A dark room can be easily illuminated with this red radiation. We have used this fact to explore a possible realization of chlorophyll laser which would be a natural environmental phenomenon.
\end{abstract}

Keywords: Chlorophyll Laser; Fluorescence

\section{Introduction}

Nearly all vegetations - from the tiny grasses on the surface to the lofty trees in the forest exhibit green as its prominent color which is undoubtedly a great environmental phenomenon on our earth. Sir C. V. Raman [1,2] was particularly concerned with investigations related to the colors exhibited by foliage and by flowers in vivo. These are the colors which are actually perceived, and their relationship to the spectral character of the light reaching our eyes is the subject of study in case of Sir C. $\mathrm{V}$. Raman. Raman described in a most elegant and simplified manner the physical process involved. Sunlight is incident on the leaves of growing vegetation or on the petals of flowers. It enters the material and re-emerges after internal reflections (or diffusions) or scattering. It also may be accompanied by light which is reflected or diffused at the surface of the leaves or petals. Usually, they are not important, and their effect may be minimized by an appropriate choice of the direction of observation. They may be completely avoided if the light which emerges after passing through the flower is examined through a Hand Spectroscope. The regions of the spectrum of which there is a strong absorption can be identified. This is presumably a rough estimate but the work may be supplementad by the observation through a photographic or photometric spectrometer. The usual comparison of the spectrum with the spectrum of daylight diffused by a mall white surface gives useful information. What has been observed with absorption is also true for fluorescence, which is the main topic of discussion in the present work. We know that in many cases, immersion of the crushed plant leaves or petals in a test tube or cuvette containing a suitable organic solvent such as acetone enables, the pigments responsible for their natural color to be extracted easily. The extract may then be transferred to the observation cuvette of the suitable dimension which is held against the bright source of light which may be broad or monochromatic. The spectrum of light coming through the cuvette as absorption or fluorescence radiation can be examined visually. Extensive studies carried out by the author [3] during recent years have enabled a comprehensive view to be obtained of the nature of the fluorescence or absorption spectrum, and their relationship to the absorptive or the fluorescent properties of the pigments contained in the material of the leaves or petals. We are concerned in the present work with the fluorescence spectrum of particular plant leaves which exhibit some unusual properties. We describe the experimental procedure in the following sections.

\section{Experimental}

The specimen considered for our study is known as Alocasia Culcullata Schot (local name kolia kosu). We have 
used a $30 \mathrm{~mW}$ green $(532 \mathrm{~nm})$ solid state laser source and also a 500 Watt halogen lamp. The acetone extract of the plant leaves is kept in a cuvette of suitable dimension $(1 \mathrm{~cm} \times 1 \mathrm{~cm} \times 3 \mathrm{~cm})$. The cuvette with the solution is held in front of the slit of a glass spectrograph (ASCO two-prism) having good dispersion in the visible region (3900 - 7000) $\AA$. The radiation from the source is allowed to be incident on the sample cell with the help of a short focused convex lens and the fluorescence radiation is observed in the direction perpendicular to the direction of the incident beam. The fluorescence spectrum is recorded photographically with the help of a color film (400 ASA) which is commercially available. Exposure time of the order of a minute is adequate to photograph the fluorescence spectrum. It is very easy to measure the intensity of the fluorescence bands with the help of the software called Image J. This procedure makes it a complete instrument and as good as any recording spectrometer and in some cases even better [3]. The measure of the fluorescence intensity may be easily estimated with the help a Silicon detector equipped with digital multimeter (model PD 10 OPHIR). One can also observe the fluorescence radiation emerging from the cuvette with the help a pocket spectroscope and measure the intensity of the red region of the spectrum visually. It may be noted that the silicon photodiode is connected with an optical fiber which is kept in contact with the sample cell. The results are shown in Tables $\mathbf{1}$ and $\mathbf{2}$.

Table 1. Intensity of the fluorescence radiation from the acetone extracts of the plant leaves.

\begin{tabular}{ccc}
\hline Specimens & Name & Intensity (arb. Unit) \\
\hline 1 & Mesua ferra-L & 20 \\
2 & Mentha Spicata Linn & 35 \\
3 & Azadirachta indica & 10 \\
4 & Alocasia Culcullata Schot. & 1455 \\
5 & Lawsonia syn & 5 \\
6 & Cassia fistula Linn & 50 \\
7 & Artemisia vulgaris & 16 \\
\hline
\end{tabular}

Table 2. Pocket spectroscopic observation of the fluorescence red).

\begin{tabular}{cc}
\hline Specimens & $\begin{array}{c}\text { Visual estimate of the intensity in the red } \\
\text { sector of the spectrum }\end{array}$ \\
\hline 1 & $\mathrm{w}$ \\
2 & $\mathrm{w}$ \\
3 & $\mathrm{~ms}$ \\
4 & $\mathrm{vvs}$ \\
5 & $\mathrm{~ms}$ \\
6 & $\mathrm{~ms}$ \\
7 & $\mathrm{vw}$ \\
\hline
\end{tabular}

$\mathrm{W}$ = weak, $\mathrm{ms}=$ medium strong, $\mathrm{vw}=$ very weak.

\section{Results and Discussion}

It is now appropriate to produce the results in a comprehensive manner. The acetone extract of the plant leaves Alocasia Cullata Schot exhibits very inhense fluorescence when the radiation from the source is incident on the sample tube and the entire tube acts as a secondary source of intense red radiation. A dark room is illuminated with this red light similar to the red light from a 15 watt bulb. Figure 1 shows the projected image of the light at a distance of $30 \mathrm{~cm}$. from the sample cell. This type of strong fluorescence is not seen in any of the earlier fluorescence that we have examined in case of acetone extracts of other specimens.

When photographed on the glass spectrograph the fluorescence spectrum exhibits a broad and intense band in the red sector ranging from 6000 to $6300 \AA$. Figure 2 shows the fluorescence spectrum along with the intensity distribution pattern works out with the help of the software (Image J).

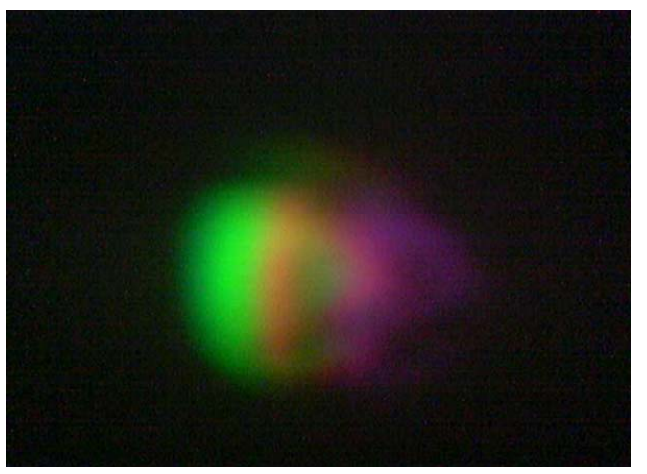

Figure 1. Projected image of the red fluorescence at a distance of $30 \mathrm{~cm}$ from the sample cell.

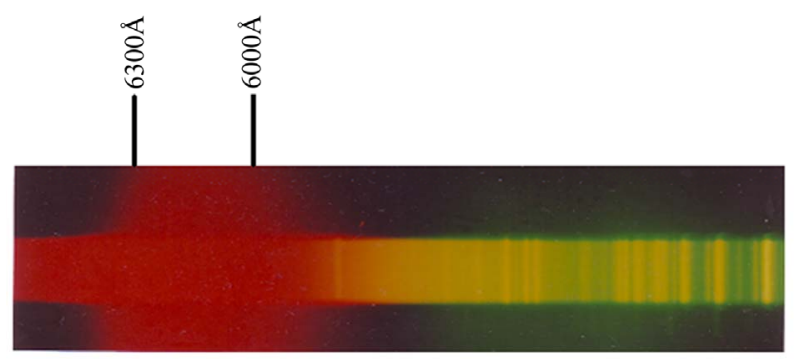

(a)

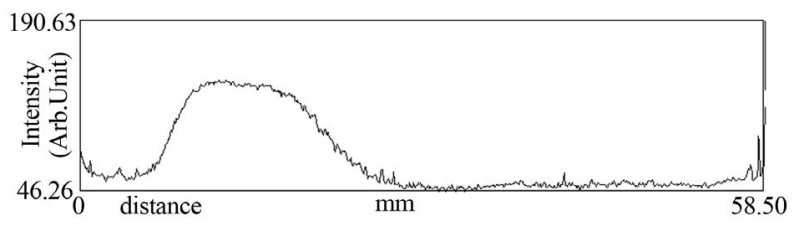

(b)

Figure 2. (a) Fluorescence spectrum of Alocasia Culcullata Schot; (b) Intensity distribution of the fluorescence bands worked out with the help of the software. 
The fluorescence originating from the acetone extracts of the leaves presumably originates from the chlorophyll a molecule. Survey of literature indicates that the fluorescence bands excited by the blue/red light consist of two bands lying at $685 \mathrm{~nm}$ and $735 \mathrm{~nm}$ (far red).

As may be inferred from Tables $\mathbf{1}$ and $\mathbf{2}$ the intensity of the fluorescence radiation originating from Alocasia culcullata schot is unbelievably strong. As for example it is more than one hundred times as compared with the intensity of the specimen number 3 . Similar is the case for other specimens. Pending further work in this field it is reasonable to believe that the high quantum yield of the fluorescence radiation of Alocassia Culcullata schot is a promising candidate for chlorophyll laser. In that case it will be a first instance of a bio-laser.

It is now appropriate to elaborate further on the nature of the fluorescence originating from the specimen. As has been emphasized earlier, such type of strong red fluorescence has not been observed from other specimens. Chlorophyll is a family of photoreceptive molecules of cynobacteria, algae and plants to perform photosynthesis. As can be seen from Figure $\mathbf{3}$ Chlorophyll molecules are largely made of carbon and hydrogen atoms and some nitrogen containing molecules surrounding magnesium atoms.

There are several kinds of Chlorophyll dominant one is chlorophyll a. Chlorophyll functions by absorbing light, which excites the electrons within the molecule and produces series of complex reactions. It may be noted that alternating single and double bonds, known as conjugated bonds, such as those in the porphyrin rings of Chlorophyll are responsible for the absorption of light in the visible region. The nature of the absorption spectra of the molecules (Chlorophyll-a and b) indicates that although absorption is there in the yellow-green sector of the spectrum the intensity is very feeble. This presumably accounts for the general green color of the leaves. From what has been discussed above we observe that the sun shines by far the brightest in the yellow green sector of the spectrum, the region where the Chlorophyll absorption is minimum. The red fluorescence is due to the Chlorophyll molecule. It is true that there are many fluorescence absorbers, but why such Chlorophyll is a good
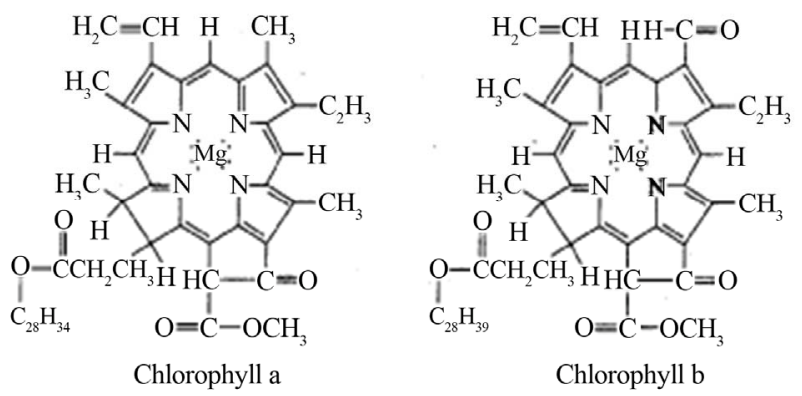

Figure 3. Structure of Chlorophyll-a and b.

candidate is still an unsolved mystery. It is not worthwhile to discuss at this stage about the expected efficiency in amplification process when such Chlorophyll is used in laser light generation but it is possible to use a cavity to investigate the strong fluorescence to know the characteristic features like coherence and gain. It is reasonable to believe that the proposed Chlorophyll laser will be comparable to the current diode sources.

\section{Conclusion}

The studies regarding the fluorescence of the acetone extracts of medicinal plant leaves have led to a new picture of the nature of fluorescence originating from chlorophyll molecule. Extremely high fluorescence yield of the specimen Alocasia Culcullata schot as compared to many other specimens indicates that it is a natural choice for the realization of a chlorophyll laser emitting radiation in the visible region.

\section{REFERENCES}

[1] C. V. Raman, "The Physiology of Vision,” The Indian Academy of Science, Bangalore, 1968.

[2] C. V. Raman, "The New Physiology of Vision-The Green color of Vegetation,” Proceedings of the Indian Academy of Science, Vol. A62, 1965, pp. 73-77.

[3] M. Konwar, "Optical and Spectroscopic Studies of Some Medicinal Plants and Natural Dyes,” Ph.D. Thesis, Dibrugarh University, Dibrugarh, 2007; Other References Regarding Chlorophyll Fluorescence May Be Found in the Thesis. 